\title{
Androgens influence expression of matrix proteins and proteolytic factors during cutaneous wound healing
}

\author{
Stephen C Gilliver ${ }^{1}$, Jayalath PD Ruckshanthi ${ }^{1}$, Susan J Atkinson ${ }^{2}$ and Gillian S Ashcroft ${ }^{1}$
}

Excessive proteolytic activity is a feature of chronic wounds such as venous ulcers, in which resolution of the inflammatory response fails and restorative matrix accumulation is delayed as a consequence. The inflammatory actions of native androgens during the healing of acute skin wounds have lately been characterized. We have now investigated the hypothesis that such activities may impact upon the balance between anabolic and catabolic processes during wound healing. We report that wound deposition of both type I collagen and fibronectin is increased in castrated rats compared with control animals. This response is accompanied by early increases and later decreases in overall wound levels of the key collagenolytic enzymes, matrix metalloproteinase (MMP)-1 and MMP-13. Moreover, the activities of MMP-2 and MMP9, two further enzymes that contribute to collagen digestion during venous ulceration, were significantly decreased in the wounds of castrated rats. Additional analyses provide evidence that androgens directly stimulate dermal fibroblast collagen production, which supports the suggestion that increased wound collagen deposition in androgen-deprived rats results from reduced matrix degradation (as opposed to enhanced matrix protein biosynthesis). Androgen-mediated dysregulation of the parallel processes of collagen deposition and turnover may underscore the delayed healing of cutaneous wounds in elderly male patients and further contribute to the increased incidence of non-healing wounds in this population.

Laboratory Investigation (2007) 87, 871-881; doi:10.1038/labinvest.3700627; published online 2 July 2007

KEYWORDS: castration; collagen; matrix metalloproteinases; wound healing

Following cutaneous injury, the damaged dermal matrix is replaced by activated fibroblasts, which migrate from the surrounding stroma into the wound space in a process mediated by the binding of fibroblast-expressed $\alpha 5 \beta 1$ integrin molecules to locally deposited fibronectin ${ }^{1}$ and stimulated by platelet-derived growth factor. ${ }^{2}$ Immature granulation tissue, formed as a result of transforming growth factor- $\beta$-stimulated fibroblast matrix protein biosynthesis, ${ }^{3}$ is cell-rich, highly vascularized and composed largely of fibronectin, ${ }^{4}$ proteoglycans ${ }^{5}$ and type III and V collagens. ${ }^{6}$ Over time, through tissue remodeling, these matrix components are gradually replaced by type I collagen, ${ }^{7}$ the most abundant collagen in uninjured skin. Newly deposited collagen fibers are cross-linked to form larger bundles and reorganized to give a more ordered structure. The ultimate product is a mature connective tissue scar, in which macroscopic structure and function are disturbed and tensile strength reduced compared to normal skin.
Making important contributions to the processes of matrix turnover and tissue remodeling are members of the matrix metalloproteinase (MMP) family of zinc-dependent proteases, which share the ability to cleave proteins of the extracellular matrix, including collagens, gelatins and casein. To date, 23 human MMPs have been cloned. Synthesized as prepro-enzymes and secreted as an inactive pro-form, they have variously been implicated in a range of physiological processes, including tissue remodeling, organ morphogenesis, angiogenesis and embryonic development, and in several pathological conditions, including cardiovascular disease, neoplasias and rheumatoid arthritis. ${ }^{8}$

Of particular current interest are the varied roles of MMPs during skin repair. ${ }^{9}$ Collagenase- 1 (MMP-1) ${ }^{10}$ and stromelysin-2 (MMP-10), ${ }^{11}$ induced in basal epidermal cells at the wound edge, facilitate keratinocyte migration and/or remodeling of the newly restored basement membrane. Gelatinase A (MMP-2) also is expressed by the epidermis

\footnotetext{
${ }^{1}$ Faculty of Life Sciences, University of Manchester, Michael Smith Building, Manchester, UK and ${ }^{2}$ Department of Oncology, Cancer Research UK Cambridge Research Institute, Li Ka Shing Centre, University of Cambridge, Cambridge, UK

Correspondence: Professor GS Ashcroft, MD, PhD, Faculty of Life Sciences, University of Manchester, Michael Smith Building, Oxford Road, Manchester M13 9PT, UK. E-mail: gillian.s.ashcroft@manchester.ac.uk
} 
following injury and, along with gelatinase B (MMP-9), ${ }^{12}$ by infiltrating macrophages. It is worth noting also that MMP-2 and MMP-9 possess elastolytic ${ }^{13}$ and fibronectin-cleaving capabilities, ${ }^{14,15}$ suggesting that neodermal elastin and fibronectin may be susceptible to degradation by locally secreted gelatinases. Controlled cleavage of provisional neomatrix constituents by MMP-2 contributes to connective tissue remodeling. ${ }^{16}$ The type I collagen of which mature scar tissue is largely composed is a substrate for digestion by gelatinases, following initial cleavage by MMP-1 or, potentially, MMP-13 and MMP- $14 .{ }^{17}$

The healing of acute skin wounds is significantly delayed in the elderly. Re-epithelialization ${ }^{18}$ and restoration of the basement membrane ${ }^{19}$ are severely retarded and local inflammation, an impediment to efficient repair, increased. ${ }^{19}$ In aged mice, moreover, delayed wound closure is accompanied by reduced local levels of fibronectin and collagens I, III and IV. ${ }^{20}$ Nonetheless, the cosmetic appearance and microscopic structure of newly synthesized scar tissue are improved in aged humans. ${ }^{21}$ However, a feature of delayed acute wound healing in the elderly is increased wound MMP-2 and MMP-9 protein levels ${ }^{12}$ and wound fluid activities of the same two proteases are markedly elevated in venous ulcer patients. ${ }^{22}$

Intriguingly, the results of neural network studies indicate that elderly male patients are at greater risk of developing venous ulceration than are their female counterparts. ${ }^{23}$ This finding parallels a similar gender divergence in the healing of acute skin wounds: restoration of the basement membrane (collagen VII accumulation) and matrix collagen deposition progress more slowly in elderly males than in age-matched female patients. ${ }^{19}$ Such observations prompted enquiry into the possible involvement of sex steroids in the regulation of repair. As a result, estrogens have been identified as being enhancers of repair that accelerate healing ${ }^{18,24}$ and androgens as inhibitors that retard the recovery from injury. ${ }^{19}$

In order more adequately to describe the ways in which androgen status impacts upon wound matrix protein profiles, and the mechanisms involved, male Sprague-Dawley rats have been used to study the effects of castration upon fibronectin, matrix collagens and MMPs with known associations to skin repair. On day 6 post-injury, wound levels of collagen I and fibronectin were increased in castrated rats compared with intact controls, despite the fact that two native androgens, testosterone and $5 \alpha$-dihydrotestosterone (DHT), increased the production of type I collagen by rat skin fibroblasts. Wound levels of the fibrillar collagenases MMP-1 and MMP-13 peaked on day 2 post-wounding in castrated rats, compared with day 6 in controls, suggesting the persistence of collagenolytic activity in intact animals, subsequently confirmed. Similarly, day 6 wound levels of proand active MMP-2 and MMP-9 were reduced through castration. These data suggest that altered proteolytic activities underscore the increased accumulation of wound matrix proteins in castrated rodents described here and previously.

\section{MATERIALS AND METHODS}

\section{Wound Healing Experiments}

Eight-week-old male Sprague-Dawley rats were anaesthetized with isoflurane and their dorsa shaved and cleansed using ethanol. Each animal received four $1 \mathrm{~cm}$ dorsal incisions through the skin and underlying panniculus carnosus muscle 5 and $10 \mathrm{~cm}$ caudal to the base of the skull and $1 \mathrm{~cm}$ either side of the midline. A subset of animals had been castrated 2 weeks before wounding. Animals were housed according to treatment group post-operatively, and wounds were excised on days $2(n=6-7$ per treatment group) and $6(n=5-6)$ post-wounding. Immediately following excision, each wound was bisected; one half was snap-frozen in liquid nitrogen and stored at $-80^{\circ} \mathrm{C}$ and the other half processed in $8 \%$ formaldehyde-based fixative for wax embedding. Isolated by means of cardiac puncture, sera were analysed for testosterone levels by enzyme immunoassay (MP Biomedicals, Cambridge, UK).

\section{Immunohistochemistry and Image Analysis}

Five-micrometer-thick histological sections prepared from wound tissue fixed in $8 \%$ formaldehyde-based fixative were embedded in paraffin. Sections prepared from the central portion of each wound were subjected to immunohistochemistry with monoclonal IgG antibodies raised against type I and III collagens (Sigma-Aldrich, Gillinghams, UK) and polyclonal antibodies raised against cellular and extracellular fibronectin and MMP-9 (Autogen Bioclear, Calne, UK); MMP-1 (Merck Biosiences, Nottingham, UK); MMP-2 (Chemicon, Chandlers Ford, UK); and MMP-1 and MMP-13 (prepared in sheep ${ }^{25,26}$ ). Sections on each slide were treated with phosphate-buffered saline instead of primary antibody as a negative control, in each case producing no positive staining. Bound primary antibody was detected using a colorimetric VECTASTAIN ABC avidin-biotin-peroxidase kit (Vector Labs, Peterborough, UK) in conjunction with the peroxidase substrate Novared (Vector Labs). Photographic images were captured and numbers of immunostained cells per unit area quantified using Image-Pro Plus software (Media Cybernetics, Marlow, UK), as described previously. ${ }^{18}$

\section{Picro-Sirius Red Staining}

Paraffin-embedded wound sections were immersed in $0.1 \%$ Sirius red (in picric acid) for $1 \mathrm{~h}$ and then washed in acidified water. When examined under plane-polarized light, larger collagen fibres appear red, orange or yellow and the thinner ones green. This birefringence is highly specific to collagen. ${ }^{27}$

\section{Quantification of Matrix Collagen and Fibronectin Levels}

Immunohistochemical and Picro-Sirius red staining intensities were compared by four-grade semiquantitative scoring: 0 , no neomatrix staining; 1 , weak staining; 2 , 
moderate staining; 3, strong staining. Data are presented as box-and-whisker plots.

\section{Fibroblast Treatment Assays}

Primary rat dermal fibroblasts were isolated using a previously described explant technique. ${ }^{28}$ Cells were grown in phenol red-free Dulbecco's modified Eagle's medium supplemented with $5 \%$ heat-inactivated fetal bovine serum. At passage five, cells were seeded to six-well plates and at approximately $90 \%$ confluence were switched to growth medium supplemented with $5 \%$ charcoal-stripped serum for a period of $24 \mathrm{~h}$. Androgen treatments (testosterone, $10 \mathrm{nM}$ or DHT, $10 \mathrm{nM}$ ) were applied for a further $72 \mathrm{~h}$.

\section{Quantitative Real-Time PCR}

Total RNA was extracted from frozen day 2 and 6 wound tissue using Trizol (Invitrogen, Paisley, UK) and was purified using RNeasy Mini kits (Qiagen, Crawley, UK) in accordance with the manufacturer's instructions. cDNA was synthesized from $1 \mu \mathrm{g}$ of wound RNA using a reverse transcription kit (Promega, Southampton, UK) in conjunction with an AMVreverse transcriptase (Roche, Lewes, UK). Quantitative realtime PCR (qPCR) analysis was performed using a SYBR Green I core kit (Eurogentec, Romsey, UK) and an Opticon qPCR thermal cycler (Genetic Research Instrumentation, Braintree, UK), following the manufacturers' guidelines. For each primer set, an optimal sample dilution was determined and melting curve analysis was used to assess the specificity of product amplification. Each test sample was serially diluted over three orders of magnitude, and all samples were analysed concurrently. PCR was performed using primer pairs designed to amplify specific sequences of the genes encoding the $\alpha 1$ and $\alpha 2$ chains of type I collagen; the $\alpha 1$ chain of type III collagen; fibronectin 1; MMP-1a, MMP-1b, MMP-2, MMP-9 and MMP-13; and the housekeeping genes which encode $18 \mathrm{~S}$ rRNA, glyceraldehyde-3-phosphate dehydrogenase, hypoxanthine guanine phosphoribosyl transferase, and tyrosine 3-monooxygenase/tryptophan 5monooxygenase activation protein, $\zeta$ (for normalization). Primer sequences are listed in Table 1.

\section{Immunoblotting}

Total protein was extracted from rat wound tissue and normal dermal fibroblasts using an SDS-based detergent buffer. Extracted protein samples ( $n=5$ per treatment group) were pooled and $1 \mathrm{mg}$ was tested as has been described previously. ${ }^{12}$ Briefly, samples separated by denaturing, reducing SDS-PAGE were blotted to $0.2 \mu \mathrm{m}$ nitrocellulose membrane (Bio-Rad, Hemel Hempstead, UK). Membranes were then blocked for $16 \mathrm{~h}$ in Tris-buffered saline supplemented with $0.1 \%$ Tween-20 and 5\% non-fat dry milk. Following sequential $1 \mathrm{~h}$ incubations with primary and horseradish

Table 1 Primer sequences and product sizes for qPCR-amplified genes

\begin{tabular}{|c|c|c|c|}
\hline Target gene & Forward primer sequence & Reverse primer sequence & Product size (bp) \\
\hline Collata & acgtcctggtgaagttggtc & tccagcaataccctgaggtc & 118 \\
\hline $\mathrm{Col} a 2^{b}$ & acctcagggtgttcaaggtg & cttctccagcggtaccagag & 95 \\
\hline $\mathrm{Col} 3 a 1^{\mathrm{c}}$ & ctggtcctgttggtccatct & acctttgtcacctcgtggac & 131 \\
\hline$F n 1^{d}$ & ggggtcacgtacctcttcaa & tggaggttagtgggagcatc & 104 \\
\hline Mmpla & gcatgcttagccttcctttg & ctgaaacacggggaaactgt & 68 \\
\hline Mmplb & gaaaccccgagtgctatcaa & tcttggcaaatgtggtgtgt & 138 \\
\hline Mmp2 & tgggggagattctcactttg & ccatcagcgttcccatactt & 87 \\
\hline Mmp9 & cactgtaactgggggcaact & agagtactgcttgcccagga & 73 \\
\hline Mmp13 & gaggtgaaaaggctcagtgc & atgaggcggggatagtcttt & 128 \\
\hline$R n r 1^{\mathrm{e}}$ & agtccctgcctttgtacaca & gatccgagggcctcactaac & 69 \\
\hline Gapdh $^{f}$ & tgccactcagaagactgtgg & ggatgcagggatgatgttct & 85 \\
\hline $\mathrm{Hprt}^{\mathrm{g}}$ & caggccagactttgttggat & ccgctgtcttttaggctttg & 146 \\
\hline$Y_{w h a z^{\mathrm{h}}}$ & ttgagcagaagacggaaggt & gaagcattggggatcaagaa & 136 \\
\hline
\end{tabular}

\footnotetext{
${ }^{\mathrm{a}}$ Encodes the $\alpha 1$ chain of type I collagen.

${ }^{b}$ Encodes the $\alpha 2$ chain of type I collagen.

'Encodes the $\alpha 1$ chain of type III collagen.

$\mathrm{d}_{\text {Encodes fibronectin } 1 .}$.

Encodes the $18 \mathrm{~S}$ ribosomal RNA.

Encodes glyceraldehyde-3-phosphate dehydrogenase.

Encodes hypoxanthine guanine phosphoribosyl transferase.

h Encodes tyrosine 3-monooxygenase/tryptophan 5-monooxygenase activation protein, $\zeta$.
} 
peroxidase-linked secondary antibodies, antigen binding was probed using ECL Plus detection reagent (GE Healthcare, Little Chalfont, UK). Primary antibodies raised against type I and III collagens, and (as a loading control) $\beta$-actin (all mouse monoclonal IgGs) (all Sigma-Aldrich); fibronectin (rabbit polyclonal IgG) (Santa Cruz); MMP-1 (Merck Biosciences, Nottingham, UK) (mouse monoclonal $\mathrm{IgG}$ ); and MMP-13 (sheep polyclonal $\mathrm{IgG}^{26}$ ) were used in conjunction with donkey anti-rabbit, sheep anti-mouse (both from GE Healthcare) and donkey anti-sheep (AbD Serotec, Oxford, UK) secondary antibodies (IgGs).

\section{Macrophage Treatment Assays}

Peritoneal macrophages were isolated by intraperitoneal lavage with ice-cold, sterile phosphate-buffered saline, followed by purification using Ficoll-Paque Plus (GE Healthcare) in accordance with the manufacturer's instructions. Cells were pooled and then diluted to a concentration of $2 \times 10^{6}$ cells $/ \mathrm{ml}$ in phenol red-free Dulbecco's modified Eagle's medium supplemented with $5 \%$ charcoal-stripped fetal bovine serum. Treatments (DHT, $10 \mathrm{nM}$ and/or bacterial lipopolysaccharide, $1 \mu \mathrm{g} / \mathrm{ml}$ ) were applied for a period of $48 \mathrm{~h}$.

\section{Collagenase Assay}

Total rat wound protein was extracted in a denaturing, nonreducing buffer (100 mM Tris, $20 \mathrm{mM} \mathrm{NaCl}, 1 \%$ (w/v) SDS, $\mathrm{pH}$ 7.6). Sample type I collagenase activities were quantified using a rapid collagenase assay kit (MD Biosciences, Zürich, Switzerland). Briefly, wound protein samples and APMAactivated MMP-8 standards were incubated with FITClabeled bovine type I collagen for $2 \mathrm{~h}$ at $35^{\circ} \mathrm{C}$. Fluorescence intensities of the resultant digested collagen fragments, separated from intact fibrils through organic solvent extraction, were measured with a $\lambda_{\text {excitation }}$ of $490 \mathrm{~nm}$ and a $\lambda_{\text {emission }}$ of $520 \mathrm{~nm}$. Sample collagenase activities were then determined from a standard curve of activated MMP- 8 values.

\section{Gelatin Zymography}

Total protein was extracted from rat wound tissue and androgen-treated macrophages using denaturing, non-reducing buffer. Thus extracted protein samples $(n=5$ per treatment group) were pooled and $50 \mu \mathrm{g}$ tested for gelatinase activities, as described previously. ${ }^{29}$ Briefly, an acrylamide gel, containing $0.5 \mathrm{mg} / \mathrm{ml}$ gelatin, was prepared. Protein samples were separated by SDS-PAGE, under non-reducing conditions and alongside human MMP-2 and MMP-9, purified from stably transfected mouse myeloma cells according to a previously described method. ${ }^{30}$ Following separation, gels were washed in $2.5 \%$ Triton X-100 (Sigma-Aldrich) and, briefly, in running $\mathrm{dH}_{2} \mathrm{O}$, before being incubated for $16 \mathrm{~h}$ at room temperature in assay buffer $(100 \mathrm{mM}$ Tris, $30 \mathrm{mM}$ $\mathrm{CaCl}_{2}, 0.02 \%(\mathrm{w} / \mathrm{v})$ sodium azide, $0.05 \%(\mathrm{v} / \mathrm{v})$ Brij 35, $\mathrm{pH}$ 7.9). They were subsequently stained with Coomassie Brilliant Blue $\mathrm{G}$ for $30 \mathrm{~min}$ and destained in $1 \%$ acetic acid, $30 \%$ methanol.

\section{Statistical Analysis}

The software package Simfit (version 5.7.4) (William Bardsley, University of Manchester, Manchester, UK) was utilized to determine statistical differences by one-way analysis of variance or unpaired, two-tailed Student's $t$-tests or, for non-parametric data, by pairwise Mann-Whitney $U$-tests (with Bonferroni correction where necessary). A $P$-value $<0.05$ was considered significant.

\section{RESULTS}

\section{The Effects of Castration upon Wound Collagenous Matrix Deposition}

A connection between systemic androgen levels and the extent of collagen deposition during wound healing was revealed by studies on castrated mice, which accumulate greater amounts of collagen than do intact littermates. ${ }^{19}$ In order to probe further the potential roles of native androgens in regulating these processes, patterns of wound collagen expression in intact and castrated rats have been examined. Corroborating the previous findings in mice, overall day 6 wound collagen accumulation, when assessed through PicroSirius red staining, was greatly increased in castrated rats (Figure 1a), in which circulating testosterone levels were reduced $>94 \%$ relative to controls (data not shown). Subsequent examination of individual collagen species by immunohistochemistry indicated that, while day 6 wound levels of type I collagen are increased in castrated animals, type III collagen is present in equivalent amounts (Figure 1a). These findings were confirmed by immunoblotting for the same two proteins, overall type I, but not type III, collagen levels in day 6 wounds from castrated rats exceeding those in intact animals (Figure 1b). The increase in the levels of type I collagen appears not to follow on from a prior increase in mRNA expression, since the mRNA species which encode the $\alpha 1$ and $\alpha 2$ chains of this protein displayed a (nonsignificant) trend towards reduced abundance in castrated animals (Figure 1c). Fibroblasts are the primary cellular source of type I collagen during wound healing. ${ }^{3}$ When dermal fibroblasts cultured from intact male rats were treated with either DHT or testosterone, the production of type I collagen was markedly increased (Figure 1d). This last finding suggests that, far from constraining wound collagen production, as might have been inferred from the increase we observed in androgen-deprived animals, androgens may directly stimulate matrix collagen production. That this apparently anabolic response does not translate into increased neodermal collagen accumulation in intact animals, relative to castrated ones, invokes the possible involvement of degradative mechanisms.

\section{Wound Fibronectin Levels are Increased in Castrated Rats}

Deposited during the initial stages of the post-injury response, fibronectin represents one of the principal components of the crude provisional wound matrix and a key 

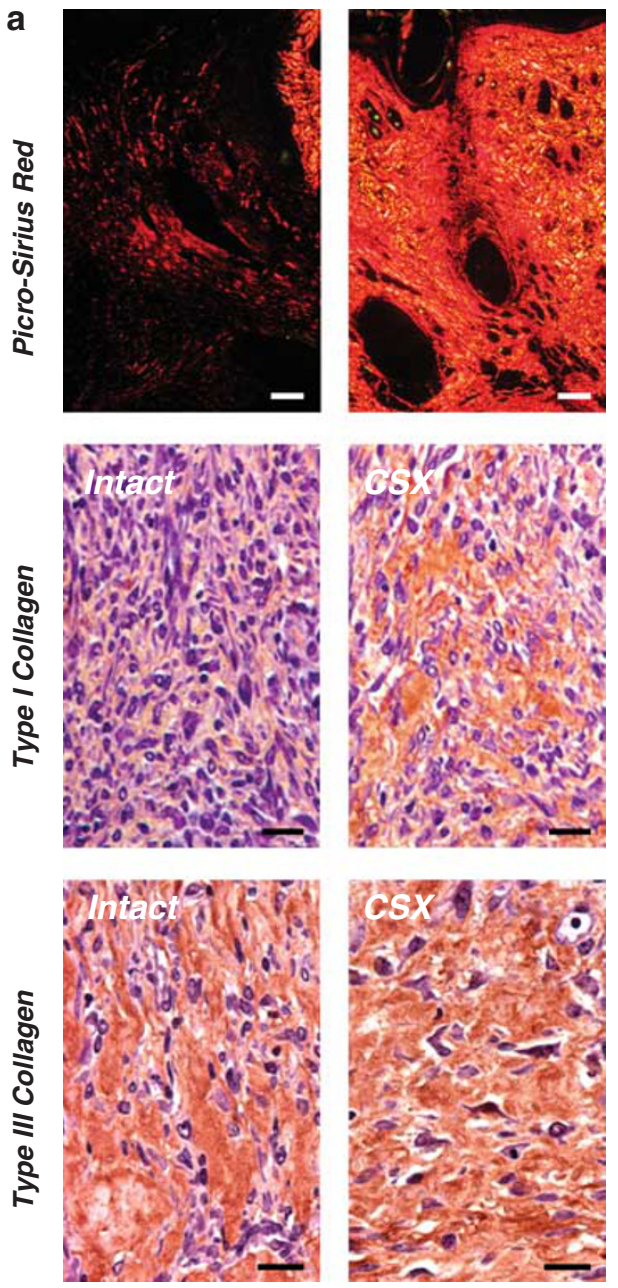

Figure 1 Wound collagen accumulation is increased as a consequence of castration. (a) Day 6 wound tissue from intact (IN) and castrated (CSX) rats was analysed for collagen content by Picro-Sirius staining (top panels) and immunohistochemical expression of collagen I (middle panels) and III (bottom panels). (a, b) Overall day 6 wound type I collagen (but not collagen III) levels were increased in castrated animals. (c) Expression of the genes encoding the type I and III collagen polypeptides was not significantly affected by castration (data expressed as mean \pm s.e.m.). Rat dermal fibroblast production of type I collagen (d) was increased as a result of treatment with DHT or testosterone (T). $n=5-6$ per treatment group. ${ }^{\star} P<0.05$ (intact vs CSX). Scale bars define a distance of $100 \mu \mathrm{M}$ (Picro-Sirius red staining) or $25 \mu \mathrm{M}$ (collagen immunostaining).

scaffold protein upon whose filaments inflammatory cells and fibroblasts immigrate. Using antibodies raised against both the intracellular and extracellular forms of fibronectin 1, the effects of castration upon wound fibronectin expression profiles were studied. While day 6 wound extracellular fibronectin levels were increased in castrated rats, levels of intracellular fibronectin, primarily localized to fibroblasts, were comparable between intact and castrated animals (Figure $2 \mathrm{a}$ and $\mathrm{b}$ ). Overall wound fibronectin protein levels in castrated rats, assessed by immunoblotting, exceeded those in intact animals (Figure 2c). Mirroring the observed pattern of expression of the mRNA species which encode type I collagen, the gene encoding fibronectin 1 exhibited a trend towards reduced day 6 wound expression in castrated rats (Figure 2d). The increase in extracellular fibronectin levels in the wounds of these animals thus apparently does not occur b
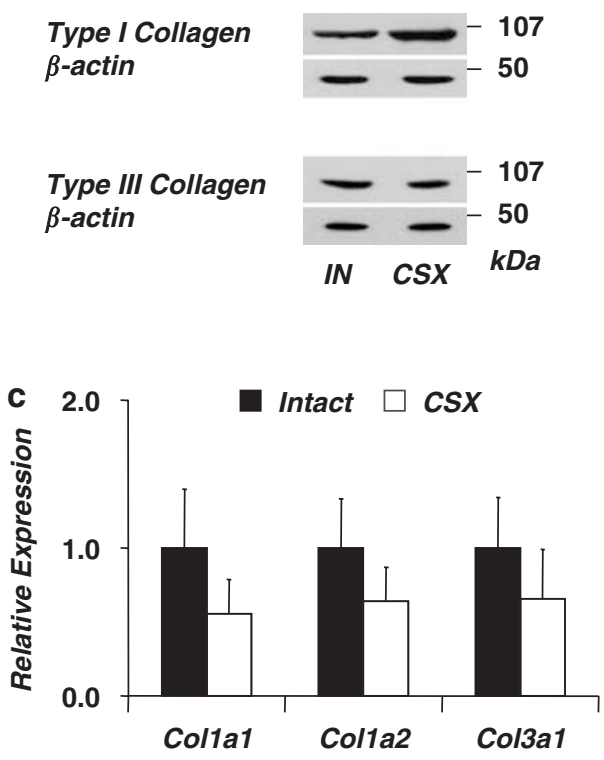

d

Type I Collagen $\beta$-actin

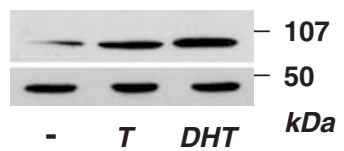

as the consequence of a direct transcriptional response. Moreover, the production by dermal fibroblasts of fibronectin, which is deposited by immigrating fibroblasts during skin wound healing, ${ }^{4}$ remained unaltered following treatment with either testosterone or DHT (Figure 2e). Nonetheless, since fibronectin makes a critical contribution to the influx of fibroblasts to sites of injury, any modulation of its production or processing may have important implications for fibroblast-mediated processes, including matrix protein biosynthesis.

\section{Castration Influences Wound Collagenase Expression Profiles}

Of the three 'classical' mammalian type I collagenases, two, MMP- ${ }^{10}$ and MMP- $13,{ }^{31}$ are injury-induced (MMP-8 was recently shown to be weakly expressed or absent in acute 

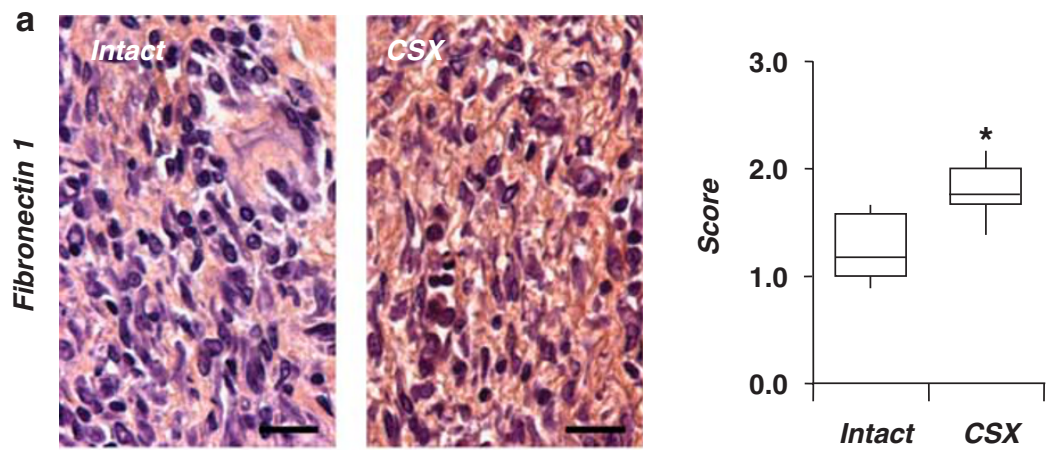

b
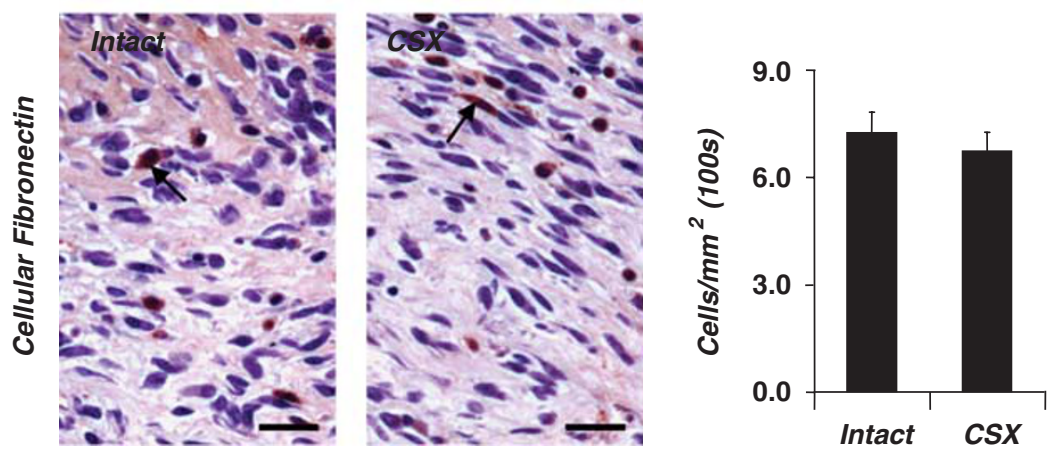
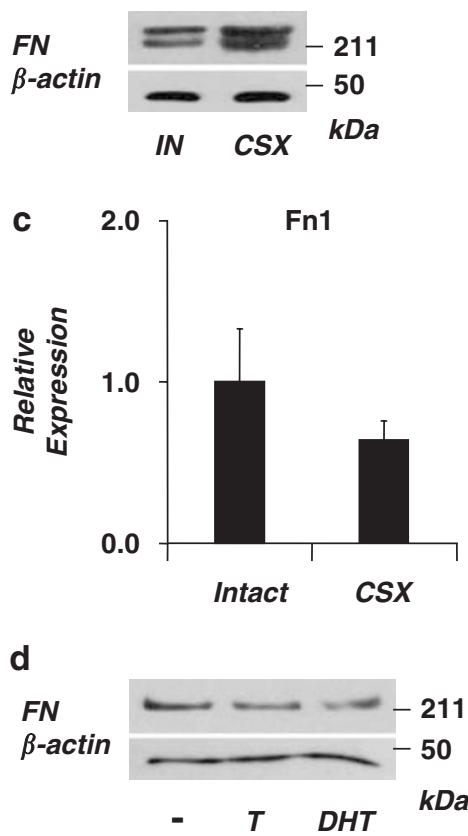

Figure 2 Wound fibronectin levels are increased in castrated rats. (a) Day 6 wound tissue from intact (IN) and castrated (CSX) rats was analysed for secreted (upper panels) and cellular fibronectin (FN) content (lower panels) by immunohistochemistry. (a, b) While numbers of fibronectin-immunoreactive cells in day 6 wounds were unaffected by castration, overall day 6 wound fibronectin levels were increased. (c) Expression of the Fn 1 gene, which encodes fibronectin was not significantly affected by castration. (d) Rat dermal fibroblast fibronectin production was unaffected by treatment with DHT or testosterone $(\mathrm{T}) . n=5-6$ per treatment group. Cell count and mRNA expression data are expressed as mean \pm s.e.m. ${ }^{\star} P<0.05$ (intact vs CSX). Scale bars define a distance of $25 \mu \mathrm{M}$. Arrows identify positively stained cells.

human wounds ${ }^{32}$ ). The potential contributions of these enzymes to wound collagenolysis have been investigated. Through immunohistochemical analyses, MMP-1 was found to localize to the neoepidermis and cells displaying a fibroblastic morphology in day 2 and 6 wounds, while MMP-13 was expressed solely by epithelial cells (Figure 3a). Overall numbers of MMP-1-immunopositive non-epidermal wound cells were similar in intact and castrated rats on days 2 and 6 post-wounding (Figure 3b). Interestingly, total wound MMP1 and MMP-13 levels (measured through immunoblotting) were increased in castrated animals on day 2 post-injury (Figure 3c) and decreased on day 6 (Figure 3d). Quantification of wound mRNA levels by qPCR revealed day 2 expression of the Mmpla gene, predicted to encode a rat ortholog of human MMP-1, to be significantly reduced by castration, while both the Mmp1b (a second hMMP-1 ortholog) and Mmp13 genes tended towards reduced expression (Figure 3e). This suggests that the increased day 2 wound collagenase levels do not result from de novo gene expression. Overall day 6 wound Mmp1a message levels were increased in castrated rats; day 6 wound Mmp13 mRNA levels tended to be decreased. Total wound collagenase activities (measured through the capacity of extracted protein samples to degrade FITC-labeled bovine type I collagen) were similar in intact and castrated animals on day 2 post-wounding but were significantly reduced in the latter on day 6 (Figure $3 \mathrm{f}$ ). Such decreases in both individual and overall type I collagenase levels in day 6 wounds may limit the turnover of freshly deposited collagen and thereby contribute to the more effective generation and maturation of neodermal tissue that occurs in castrated rats.

\section{Wound Gelatinase Activity is Decreased as a Consequence of Castration}

Denatured type I collagen fragments generated through the activities of type I collagenases such as MMP-1 are themselves substrates for a second class of collagenolytic enzymes, the gelatinases. Gelatinases A (MMP-2) and B (MMP-9) are strongly induced in response to skin wounding and are both persistently overexpressed during chronic venous ulceration. $^{22}$ The effects of surgical androgen ablation upon wound gelatinase activity have been explored. Day 2 wound MMP-2and MMP-9-positive cell numbers tended to be decreased in castrated rats (Figure $4 \mathrm{a}$ and b); statistically significant decreases occurred on day 6 post-wounding. Since local expression of the genes encoding MMP-2 and MMP-9 was unaffected by castration at either time point tested (Figure 4c), an altered transcriptional response does not apparently account for the observed protein profiles. Gelatin zymography confirmed day 6 wound MMP-2 and MMP-9 

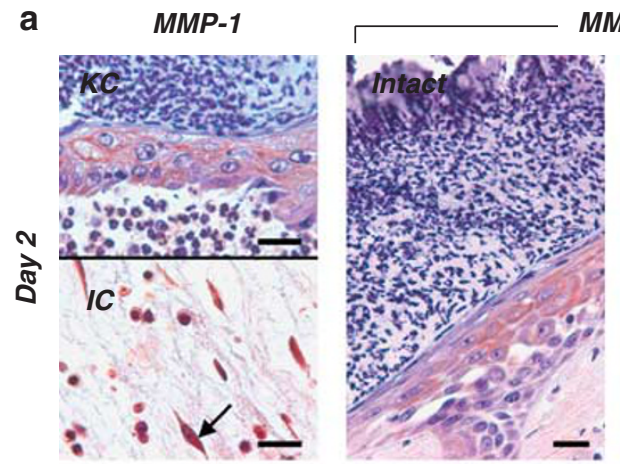

MMP-13
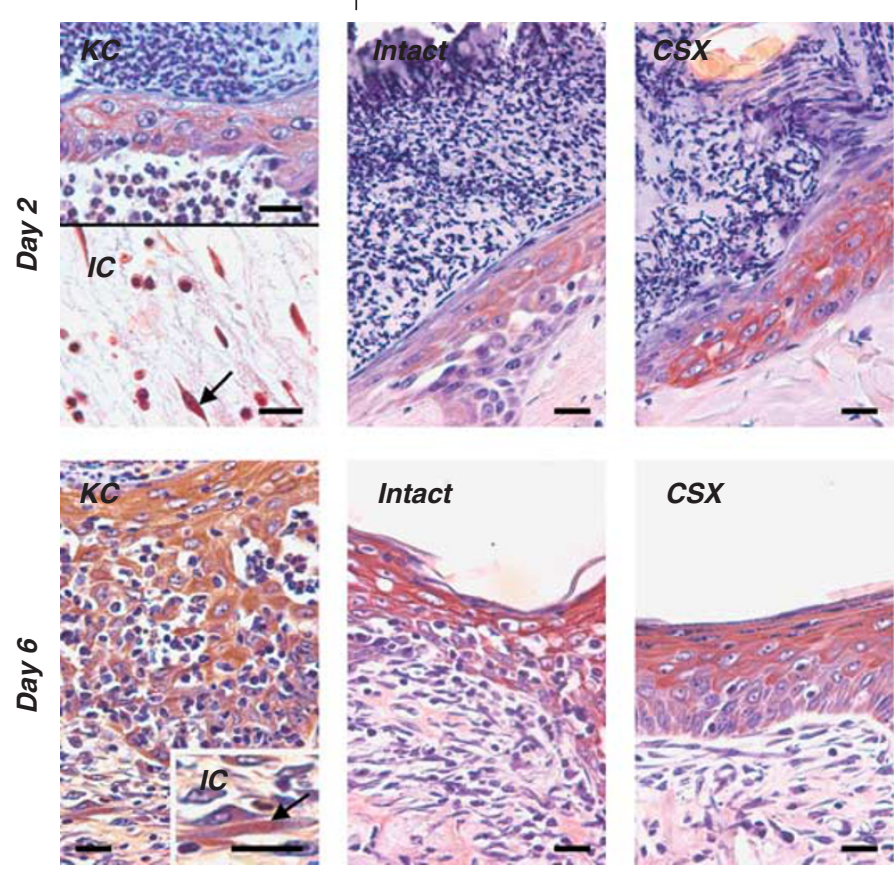
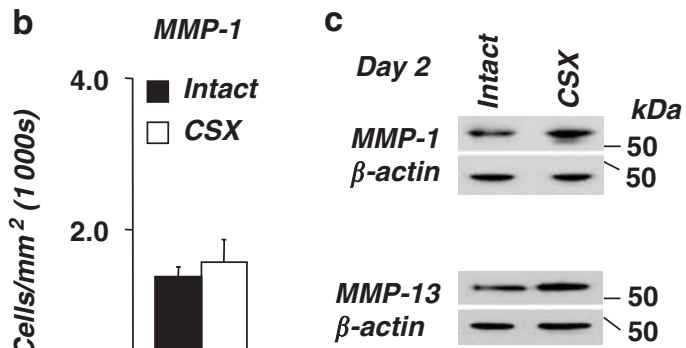

Day 2

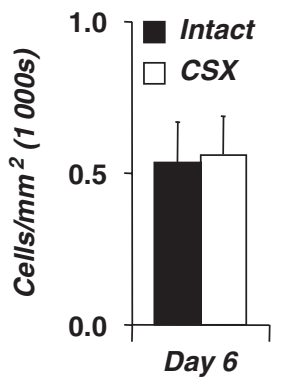

d

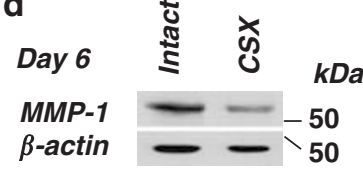

MMP-13

$\beta$-actin

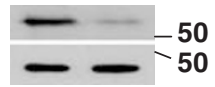

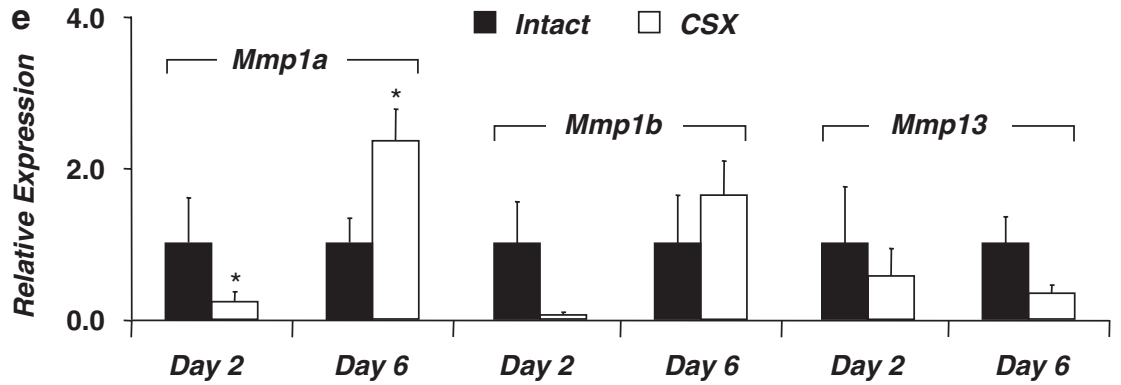

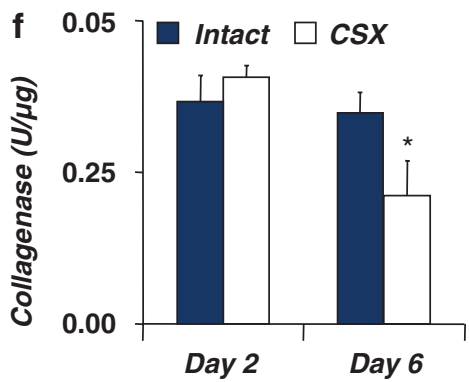

Figure 3 Wound collagenase expression peaks earlier in castrated rats. (a) Day 2 and 6 wound tissue from intact and castrated rats was analysed immunohistochemically for MMP-1 (upper panels) and MMP-13 (lower panels) protein expression profiles. MMP-1 was highly expressed by epidermal keratinocytes (KC) and infiltrating inflammatory cells (IC) in day 2 and 6 wounds in intact and castrated animals (intact shown); MMP-13 solely by keratinocytes. (b) Overall numbers of day 2 and 6 wound MMP-1-immunoreactive non-epithelial cells were unaffected by castration. (c, d) In castrated rats, overall wound MMP-1 and MMP-13 protein levels were increased on day 2 post-wounding and decreased on day 6 . (e) Expression of the genes, which encode both MMP-1 and MMP-13 tended to be reduced in day 2 wound tissue from castrated animals (in the case of the Mmp1a gene, the decrease was statistically significant). (f) Overall wound collagenase activity (in units (U) per $\mu \mathrm{g}$ of total wound protein, where $1 \mathrm{U}$ represents the cleavage of $1 \mu \mathrm{g}$ of collagen per minute) was significantly decreased in castrated animals on day 6 post-wounding. $n=5-7$ per treatment group. Data are expressed as mean \pm s.e.m. ${ }^{\star} P<0.05$ (intact $v s$ CSX). Scale bars define a distance of $25 \mu \mathrm{M}$. Arrows identify positively stained cells.

enzymatic activities to be decreased in castrated animals and revealed also that overall wound pro-MMP-9 levels were decreased on day 2 post-wounding (Figure $4 \mathrm{~d}$ ). By restricting the capacity for fibrillar collagen turnover, these decreases in injury site gelatinase activities in castrated rats may contribute to the increased collagen accumulation. Conversely, androgens may impede repair by reinforcing wound gelatinase induction. Such induction may not occur directly: the production by peritoneal macrophages of neither MMP-2 nor MMP-9 was influenced by DHT treatment (Figure 4e).

\section{DISCUSSION}

During the recovery from cutaneous injury, dermal integrity must be re-established. Wound contraction serves to limit the volume of neodermal tissue that must be deposited in order to replace that which is missing and damaged. This neodermal tissue initially consists of type III and V collagens, fibronectin and proteoglycans, which are slowly replaced by collagen I (the most abundant collagen in uninjured dermis), itself gradually remodeled as the resultant scar tissue matures. In chronic, non-healing wounds, these processes are disturbed and excessive tissue breakdown results in the failure to 

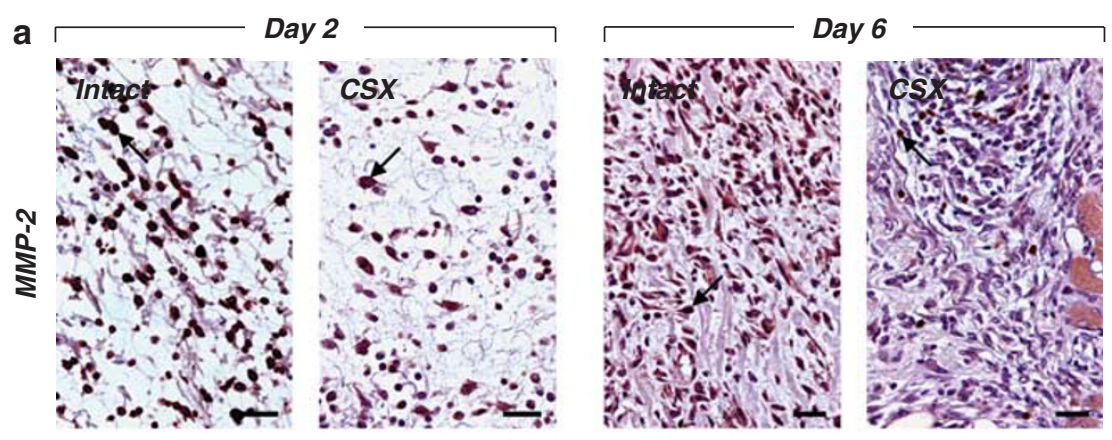

b
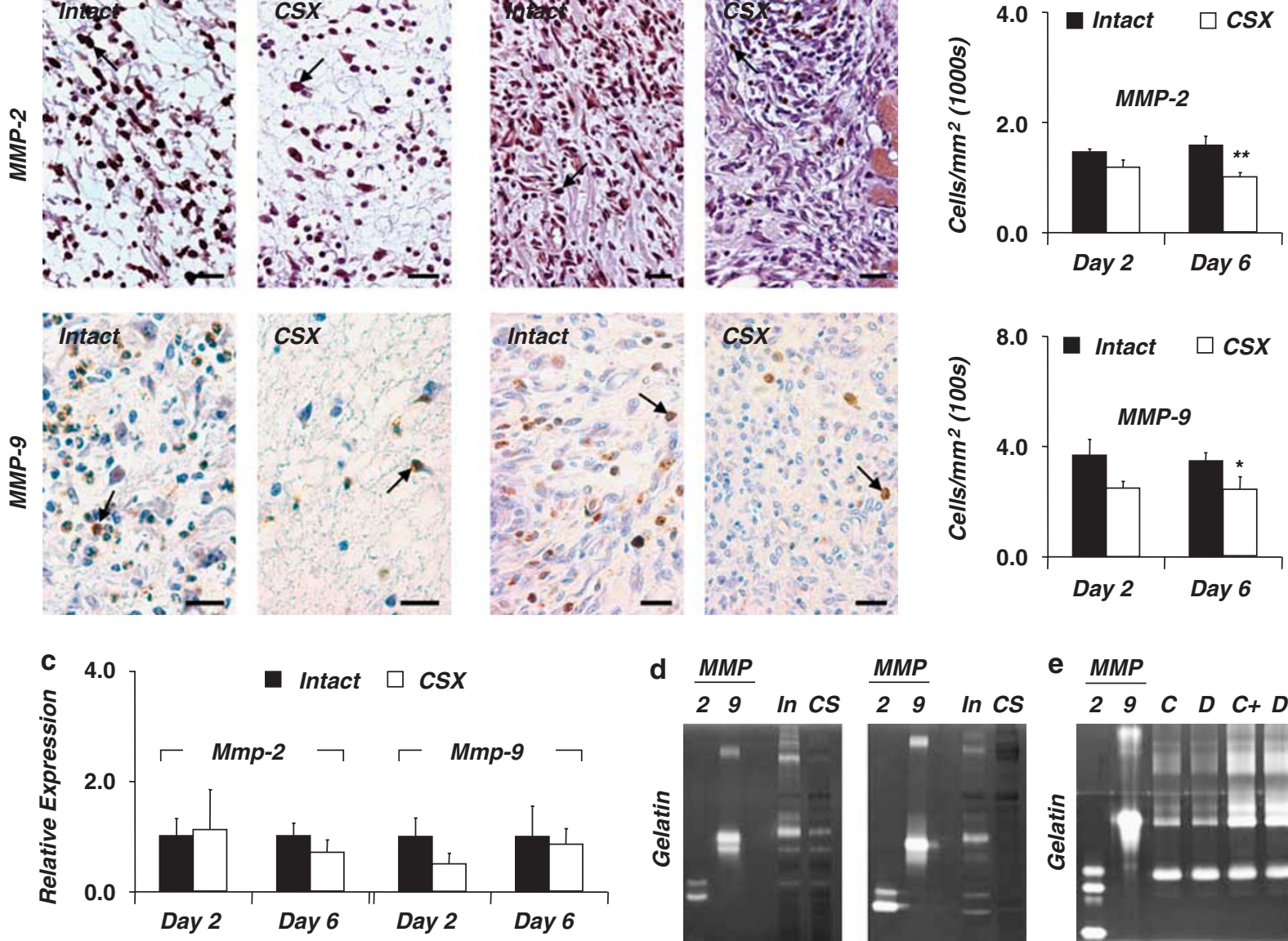

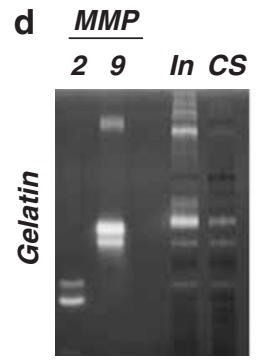

Day 2

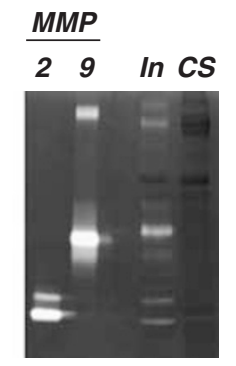

Day 6

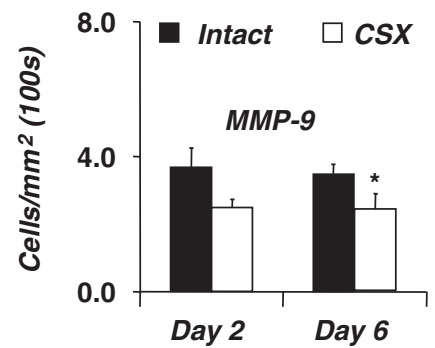

e $M M P$

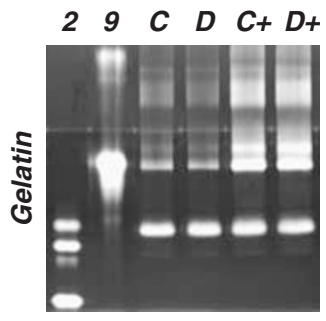

Figure 4 Castrated rats exhibit decreased wound gelatinase activity. (a) Day 2 and 6 wound tissue from (IN) intact and castrated (CSX) rats was analysed for MMP-2 (upper panels) and MMP-9 (lower panels) protein levels by immunohistochemistry. (b) Numbers of cells staining for MMP-2 and MMP-9 in day 2 wounds were not significantly affected by castration; numbers staining for MMP-2 and MMP-9 were decreased in the day 6 wounds of castrated rats. (c) Wound expression of the genes which encode MMP-2 and MMP-9 was unaffected by castration. (d) Day 2 wound pro-MMP-9 levels were decreased in castrated (CS) rats relative to intact (In) controls. MMP-2 and MMP-9 protein activity in day 6 wounds was decreased in castrated animals. (e) Macrophage gelatinase production was similar between untreated (C) and DHT-treated (D) cells, either unactivated or lipopolysaccharide-activated $(+)$. $n=5-7$ per treatment group. Data are expressed as mean \pm s.e.m. ${ }^{\star} P<0.05$, ${ }^{* *} P<0.01$ (intact vs CSX). Scale bars define a distance of $25 \mu \mathrm{M}$. Arrows identify positively stained cells.

accumulate sufficient amounts of several key matrix components as a result of both decreased rates of protein biosynthesis $^{33}$ and greatly increased proteolytic activity. ${ }^{34}$ Intriguingly, male gender is the primary factor predisposing to chronic wound healing states in humans, ${ }^{23}$ and our recent studies have demonstrated that androgens impair healing in mouse and human models. ${ }^{19}$

Previous studies have suggested that androgens may stimulate collagen production: DHT was shown to drive cutaneous expression of the gene encoding pro- $\alpha 1$ (I) collagen $^{35}$ and dermal collagen content was reported to be increased in elderly women receiving androgen treatment for osteoporosis. ${ }^{36}$ Accelerated healing in castrated mice is associated with increased local levels of matrix collagen: when analysed through Picro-Sirius staining and hydroxyproline content, overall collagen abundance in day 5 and 21 wounds was significantly increased in castrated animals compared with intact controls. ${ }^{19}$ We therefore investigated the mechanisms underlying these observations.

We have shown that overall day 6 wound type I collagen levels are significantly increased in castrated rats, which suggests that repair and remodeling of the dermis progress more rapidly in the absence of androgens. The subsequent discovery that testosterone and DHT directly stimulate the production of type I collagen protein by rat dermal fibroblasts, in addition to our observation of fewer numbers of fibroblasts per unit area in the wounds of castrated rodents (unpublished data), suggests that this response does not result from the prevention of androgen-mediated inhibition of local collagen biosynthesis during wound healing. The 
possibility that altered protease profiles might instead underpin the increase in wound collagen levels was therefore investigated.

While the role of MMP-13 during cutaneous wound healing is yet to be confirmed, MMP-1 facilitates migration of leading edge keratinocytes across the underlying stroma. ${ }^{10}$ However, persistent or ectopic collagenase expression has the potential to interfere with normal wound matrix generation. Both MMP-1 and MMP-13 are abundantly expressed in chronic, non-healing venous ulcers, in which excessive proteolysis prevents the translation of de novo collagen biosynthesis into increased protein accumulation. ${ }^{37}$ We have shown that total wound levels of both of these collagenase enzymes are modestly increased in castrated rats on day 2 post-wounding and greatly reduced on day 6 (these latter decreases being coincidental with the observed increase in local collagen levels and decrease in overall collagenase activities), suggesting that wound collagenase activities peak earlier in the absence of gonadal androgens. MMP-1 was strongly expressed by wound fibroblasts and its sustained secretion by these cells would have implications for the wound collagenous matrix integrity, since it catalyses the initial step in the degradation of type I collagen. ${ }^{17}$ Likewise, prolonged production of MMP-1 and/or MMP-13 by epidermal keratinocytes may contribute to disrupted restoration of the papillary dermis and/or basement membrane, the latter of whose key structural component collagens, types IV and VII, are substrates for MMP- $13^{38}$ and MMP-1, respectively. ${ }^{39}$

Fibrillar collagen fragments (gelatins) generated as a result of the degradative activities of MMP-1 and MMP-13 are substrates for further digestion by a second subfamily of collagenolytic MMPs, the gelatinases, two members of which, MMP-2 and MMP-9, have been implicated in the pathology of chronic venous ulceration ${ }^{22}$ and in delayed acute wound healing in the elderly, ${ }^{12}$ in whom the deposition of type I collagen is markedly reduced. ${ }^{18,24}$ Additional substrates of these enzymes include type IV collagen, elastin and proteoglycans. ${ }^{40}$ We have here shown that wound activities of MMP-2 and MMP-9, both strongly expressed by inflammatory cells on day 2 and 6 post-injury, are downregulated in castrated animals: overall levels of pro-MMP-9 were decreased in day 2 wounds; pro-MMP-2, pro-MMP-9 and active MMP-2 and MMP-9 in day 6 wounds. These responses, and those involving MMP-1 and MMP-13, suggest that androgens may influence the balance that exists between wound proteolytic and antiproteolytic factors, and that castration shifts this balance in a manner that encourages the accumulation of matrix proteins.

Such a conclusion is supported by the additional finding that day 6 wound fibronectin levels were increased in castrated animals. Although ultimately replaced by type I and III collagens, fibronectin plays a vital role in facilitating the migration of keratinocytes ${ }^{41}$ and fibroblasts, ${ }^{1}$ and studies have highlighted the beneficial effects of the topical admin- istration of fibronectin during repair. ${ }^{42,43}$ Increased wound expression of fibronectin in castrated rats may contribute to the marked enhancement of repair in such animals. Moreover, it may be linked directly to the parallel decreases in wound gelatinase activities, since fibronectin has been identified as a substrate of both MMP- $2{ }^{14}$ and MMP-9. ${ }^{15}$ In venous ulceration, the serine protease neutrophil elastase contributes to the uncontrolled degradation of wound bed fibronectin. ${ }^{44}$ Although not investigated, altered elastase activity profiles may contribute also to the pattern of fibronectin deposition we presently describe.

The mechanisms behind the observed changes in MMP expression remain to be determined and may be complex. Our data showing that castration reduces overall day 6 wound levels of MMP-1 and MMP-13 suggest that androgens may act to stimulate the expression of these two proteases. In an alternative context (prostate cancer LNCaP cells), MMP13 was reported to be androgen-inducible; ${ }^{45}$ expression of MMP-1 was, by contrast, shown to be DHT-repressed in a mechanism involving sequestration by the ligand-activated androgen receptor of the transcription factor ERM, which otherwise activates the MMP-1 promoter. ${ }^{46}$ Wound MMP-2 and MMP-9 activities on day 6 post-injury were reduced in castrated animals, suggesting that, following wounding, androgens stimulate local gelatinase activity. While few published data pertain to the regulation by androgens of MMP-9, the expression of MMP-2 was reported to be DHT- and (synthetic androgen) R1881-induced in adipose tissue ${ }^{47}$ and LNCaP cells, respectively. ${ }^{48}$ Moreover, this latter response is effected by binding of the activated androgen receptor to a pair of androgen-response elements within the MMP-2 promoter. ${ }^{49}$

These reports suggest that the downregulation of day 6 wound MMP-13 and MMP-2 protein levels we have observed in castrated rats may result from the removal of an inductive androgen signal. However, the results of our qPCR analyses (which revealed parallel, nonsignificant decreases in mean day 6 wound MMP-2, MMP-9 and MMP-13 mRNA levels) do not conclusively support the hypothesis that such responses are effected via transcriptional mechanisms. Moreover, of the two genes that putatively encode the rat equivalents of hMMP-1, one (Mmpla) displays markedly increased day 6 wound expression in castrated rats. This last finding suggests that (1) the coincidental decrease in MMP-1 protein levels arises from regulation at the post-transcriptional level-an effect upon either mRNA translation or protein stability/turnover; and (2) androgens regulate expression of the related collagenases MMP- 1 and MMP-13 by fundamentally different mechanisms. That DHT did not influence production by macrophages, either activated or unactivated, of active and inactive MMP-2 and MMP-9 suggests that, if androgens do modulate the activities of these two proteases in vivo in this cell type, the primary producer of gelatinases during skin wound healing, ${ }^{12}$ they do so indirectly through factors derived from alternative sources. One 
candidate effector is membrane type 1 (MT1)-MMP, which is wound-induced, ${ }^{50}$ activates pro-MMP- $2,{ }^{51}$ and itself directly degrades type I collagen. ${ }^{52}$ Altered MT1-MMP levels in the wounds of castrated rats may contribute to the decreased activation of MMP-2 and increased levels of collagen I in these animals.

In summary, we have identified increased proteolysis as being a likely contributory factor in androgen-impaired skin wound healing. Suppression of wound collagenase and gelatinase activities in castrated rats was associated with markedly increased accumulation of the key matrix proteins type I collagen and fibronectin, the production of which by fibroblasts is not apparently directly repressed by androgens. Given the recognized contribution of uncontrolled proteolysis to the failure to resolve repair in conditions of chronic wound healing, the present data suggest that the negative influence of androgens may be of particular clinical relevance under circumstances in which the activities of natural protease inhibitors are diminished. They pose implications also for the potential impact of androgen manipulations upon remodeling of the wound neomatrix, which, not presently studied, warrants future investigation.

\section{ACKNOWLEDGEMENT}

This research was supported by Wellcome Trust Senior Fellowship in Clinical Science Grant no. GR064256MA.

\section{DISCLOSURE/DUALITY OF INTEREST}

None.

1. Greiling D, Clark RA. Fibronectin provides a conduit for fibroblast transmigration from collagenous stroma into fibrin clot provisional matrix. J Cell Sci 1997;110:861-870.

2. Seppa H, Grotendorst G, Seppa S, et al. Platelet-derived growth factor is chemotactic for fibroblasts. J Cell Biol 1982;92:584-588.

3. Roberts $A B$, Sporn MB, Assoian RK, et al. Transforming growth factor type $\beta$ : rapid induction of fibrosis and angiogenesis in vivo and stimulation of collagen formation in vitro. Proc Natl Acad Sci USA 1986;83:4167-4172.

4. Grinnell F, Billingham RE, Burgess L. Distribution of fibronectin during wound healing in vivo. J Invest Dermatol 1981;76:181-189.

5. Yeo TK, Brown L, Dvorak HF. Alterations in proteoglycan synthesis common to healing wounds and tumors. Am J Pathol 1991;138: 1437-1450.

6. Betz P, Nerlich A, Wilske J, et al. Analysis of the immunohistochemical localization of collagen type III and V for the time-estimation of human skin wounds. Int J Legal Med 1993;105:329-332.

7. Bailey AJ, Bazin S, Sims TJ, et al. Characterization of the collagen of human hypertrophic and normal scars. Biochim Biophys Acta 1975;405:412-421.

8. Nagase H, Woessner Jr JF. Matrix metalloproteinases. J Biol Chem 1999;274:21491-21494.

9. Toy LW. Matrix metalloproteinases: their function in tissue repair. J Wound Care 2005;14:20-22.

10. Inoue M, Kratz G, Haegerstrand A, et al. Collagenase expression is rapidly induced in wound-edge keratinocytes after acute injury in human skin, persists during healing, and stops at re-epithelialization. J Invest Dermatol 1995;104:479-483.

11. Rechardt $\mathrm{O}$, Elomaa $\mathrm{O}$, Vaalamo $\mathrm{M}$, et al. Stromelysin-2 is upregulated during normal wound repair and is induced by cytokines. J Invest Dermatol 2000;115:778-787.

12. Ashcroft GS, Herrick SE, Tarnuzzer RW, et al. Age-related differences in the temporal and spatial regulation of matrix metalloproteinases
(MMPs) in normal skin and acute cutaneous wounds of healthy humans. Cell Tissue Res 1997;290:581-591.

13. Shipley JM, Doyle GA, Fliszar CJ, et al. The structural basis for the elastolytic activity of the $92-\mathrm{kDa}$ and $72-\mathrm{kDa}$ gelatinases. Role of the fibronectin type II-like repeats. J Biol Chem 1996;271:4335-4341.

14. Watanabe $\mathrm{K}$, Takahashi $\mathrm{H}$, Habu $\mathrm{Y}$, et al. Interaction with heparin and matrix metalloproteinase 2 cleavage expose a cryptic anti-adhesive site of fibronectin. Biochemistry 2000;39:7138-7144.

15. Zhao YG, Xiao AZ, Newcomer RG, et al. Activation of pro-gelatinase $B$ by endometase/matrilysin-2 promotes invasion of human prostate cancer cells. J Biol Chem 2003;278:15056-15064.

16. Agren MS. Gelatinase activity during wound healing. Br J Dermatol 1994;131:634-640.

17. Chung L, Dinakarpandian D, Yoshida N, et al. Collagenase unwinds triple-helical collagen prior to peptide bond hydrolysis. EMBO J 2004:23:3020-3030.

18. Ashcroft GS, Dodsworth J, van Boxtel E, et al. Estrogen accelerates cutaneous wound healing associated with an increase in TGF- $\beta 1$ levels. Nat Med 1997;3:1209-1215.

19. Ashcroft GS, Mills SJ. Androgen receptor-mediated inhibition of cutaneous wound healing. J Clin Invest 2002;110:615-624.

20. Ashcroft GS, Horan MA, Ferguson MW. Aging is associated with reduced deposition of specific extracellular matrix components, an upregulation of angiogenesis, and an altered inflammatory response in a murine incisional wound healing model. J Invest Dermatol 1997;108:430-437.

21. Ashcroft GS, Kielty CM, Horan MA, et al. Age-related changes in the temporal and spatial distributions of fibrillin and elastin mRNAs and proteins in acute cutaneous wounds of healthy humans. J Pathol 1997;183:80-89.

22. Wysocki AB, Staiano-Coico L, Grinnell F. Wound fluid from chronic leg ulcers contains elevated levels of metalloproteinases MMP-2 and MMP9. J Invest Dermatol 1993;101:64-68.

23. Taylor RJ, Taylor AD, Smyth JV. Using an artificial neural network to predict healing times and risk factors for venous leg ulcers. J Wound Care 2002;11:101-105.

24. Ashcroft GS, Greenwell-Wild T, Horan MA, et al. Topical estrogen accelerates cutaneous wound healing in aged humans associated with an altered inflammatory response. Am J Pathol 1999;155:1137-1146.

25. Hembry RM, Murphy G, Cawston TE, et al. Characterization of a specific antiserum for mammalian collagenase from several species: immunolocalization of collagenase in rabbit chondrocytes and uterus. J Cell Sci 1986;81:105-123.

26. Knauper V, Will H, Lopez-Otin $\mathrm{C}$, et al. Cellular mechanisms for human procollagenase-3 (MMP-13) activation. Evidence that MT1-MMP (MMP14) and gelatinase a (MMP-2) are able to generate active enzyme. J Biol Chem 1996;271:17124-17131.

27. Junqueira LCU, Bignolas G, Brentani RR. Picrosirius staining plus polarization microscopy, a specific method for collagen detection in tissue sections. Histochem J 1979:11:447-455.

28. Freshney RI. Culture of Animal Cells-A Manual of Basic Technique. Alan R Liss: New York, 1987.

29. Heussen C, Dowdle EB. Electrophoretic analysis of plasminogen activators in polyacrylamide gels containing sodium dodecyl sulfate and copolymerized substrates. Anal Biochem 1980;102:196-202.

30. Murphy G, Willenbrock F, Ward RV, et al. The C-terminal domain of $72 \mathrm{kDa}$ gelatinase $\mathrm{A}$ is not required for catalysis, but is essential for membrane activation and modulates interactions with tissue inhibitors of metalloproteinases. Biochem J 1992;283:637-641.

31. Wu N, Jansen ED, Davidson J. Comparison of mouse matrix metalloproteinase 13 expression in free-electron laser and scalpel incisions during wound healing. J Invest Dermatol 2003;121:926-932.

32. Pirilä E, Korpi JT, Korkiamäki T, et al. Collagenase-2 (MMP-8) and matrilysin-2 (MMP-26) expression in human wounds of different etiologies. Wound Repair Regen 2007;15:47-57.

33. Tarlton JF, Bailey AJ, Crawford E, et al. Prognostic value of markers of collagen remodeling in venous ulcers. Wound Repair Regen 1999;7:347-355.

34. Weckroth $\mathrm{M}$, Vaheri $\mathrm{A}$, Lauharanta J, et al. Matrix metalloproteinases, gelatinase and collagenase, in chronic leg ulcers. J Invest Dermatol 1996;106:1119-1124.

35. Opas EE, Gentile MA, Rossert JA, et al. Parathyroid hormone and prostaglandin E2 preferentially increase luciferase levels in bone of 
mice harboring a luciferase transgene controlled by elements of the pro- $\alpha 1(\mathrm{I})$ collagen promoter. Bone 2000;26:27-32.

36. Black MM, Shuster S, Bottoms E. Osteoporosis, skin collagen and androgen. Br Med J 1970;4:773-774.

37. Vaalamo M, Mattila L, Johansson $\mathrm{N}$, et al. Distinct populations of stromal cells express collagenase-3 (MMP-13) and collagenase-1 (MMP1) in chronic ulcers but not in normally healing wounds. J Invest Dermatol 1997;109:96-101.

38. Knäuper V, Cowell S, Smith B, et al. The role of the C-terminal domain of human collagenase-3 (MMP-13) in the activation of procollagenase3 , substrate specificity, and tissue inhibitor of metalloproteinase interaction. J Biol Chem 1997;272:7608-7616.

39. Seltzer JL, Eisen AZ, Bauer EA, et al. Cleavage of type VII collagen by interstitial collagenase and type IV collagenase (gelatinase) derived from human skin. J Cell Biol 1989;264:3822-3826.

40. Murphy G, Cockett Ml, Ward RV, et al. Matrix metalloproteinase degradation of elastin, type IV collagen and proteoglycan. A quantitative comparison of the activities of $95 \mathrm{kDa}$ and $72 \mathrm{kDa}$ gelatinases, stromelysins- 1 and -2 and punctuated metalloproteinase (PUMP). Biochem J 1991;277:277-279.

41. Takashima A, Billingham RE, Grinnell F. Activation of rabbit keratinocyte fibronectin receptor function in vivo during wound healing. J Invest Dermatol 1986;86:585-590.

42. Lariviere B, Rouleau M, Picard S, et al. Human plasma fibronectin potentiates the mitogenic activity of platelet-derived growth factor and complements its wound healing effects. Wound Repair Regen 2003;11:79-89.

43. Kwon AH, Qiu Z, Hiraon Y. Effect of plasma fibronectin on the incisional wound healing in rats. Surgery 2007;141:254-261.
44. Herrick S, Ashcroft G, Ireland G, et al. Up-regulation of elastase in acute wounds of healthy aged humans and chronic venous leg ulcers are associated with matrix degradation. Lab Invest 1997;77:281-288.

45. Pang ST, Flores-Morales A, Skoog L, et al. Regulation of matrix metalloproteinase 13 expression by androgen in prostate cancer. Oncol Rep 2004;11:1187-1192.

46. Schneikert J, Peterziel H, Defossez P-A, et al. Androgen receptor-Ets protein interaction is a novel mechanism for steroid hormonemediated down-modulation of matrix metalloproteinase expression. J Biol Chem 1996;271:23907-23913.

47. Bolduc C, Larose M, Yoshioka M, et al. Effects of dihydrotestosterone on adipose tissue measured by serial analysis of gene expression. J Mo Endocrinol 2004;33:429-444.

48. Liao X, Thrasher JB, Pelling J, et al. Androgen stimulates matrix metalloproteinase-2 expression in human prostate cancer. Endocrinology 2003;144:1656-1663.

49. Li BY, Liao XB, Fujito A, et al. Dual androgen-response elements mediate androgen regulation of MMP-2 expression in prostate cancer cells. Asian J Androl 2007;9:41-50.

50. Madlener M, Parks WC, Werner S. Matrix metalloproteinases (MMPs) and their physiological inhibitors (TIMPs) are differentially expressed during excisional skin wound repair. Exp Cell Res 1998:242:201-210.

51. Will H, Atkinson SJ, Butler GS, et al. The soluble catalytic domain of membrane type 1 matrix metalloproteinase cleaves the propeptide of progelatinase $\mathrm{A}$ and initiates autoproteolytic activation. Regulation by TIMP-2 and TIMP-3. J Biol Chem 1996;271:17119-17123.

52. Atkinson SJ, Patterson ML, Butler MJ, et al. Membrane type 1 matrix metalloproteinase and gelatinase A synergistically degrade type 1 collagen in a cell model. FEBS Lett 2001;491:222-226. 